\title{
Rating-Verfahren in der Therapieplanung bei kognitiven Kommunikationsstörungen
}

\section{The Necessity for Assessing Rating Procedures in the Treatment Planning Process in Cognitive Communication Disorders}

\author{
Frank Regenbrecht, Ralf Glindemann, Julia Büttner-Kunert
}

\begin{abstract}
Um Veränderungen in den kommunikativen Fähigkeiten abzubilden, sind Einschätzungen aus unterschiedlichen Perspektiven (vom Fachpersonal, von Betroffenen und von Angehörigen) erforderlich. Anhand eines Fallbeispiels werden im vorliegenden Beitrag drei zentrale Argumente für den Einsatz von Rating-Verfahren (BEKOS und LCQ) bei kognitiven Kommunikationsstörungen herausgearbeitet.
\end{abstract}

\section{LERNZIEL}

Der Beitrag zeigt auf, dass eine multiperspektivische Bewertung von Störungen im Gesprächsverhalten notwendig ist, um Veränderungen in den kommunikativen Fähigkeiten nach SchädelHirn-Trauma zuverlässig abzubilden.

\section{Komplexität kognitiver Kommunikationsstörungen}

Dass sprachpathologische Symptome wie eine fehlerhafte Wortwahl, morphologische Abweichungen oder Fehler in der syntaktischen Organisation sinnvoller Einheiten den kommunikativen Erfolg von Äußerungen gefährden können, ist naheliegend und unbestritten. Ebenso ist es nicht trivial, auf welche Weise auch kognitive Symptome den Kommunikationsprozess stören können.

Welche Bedingungen müssen also in sozialen Interaktionen oder bei der Verarbeitung schriftlicher Texte etwa hinsichtlich komplexer Aufmerksamkeits- oder Gedächtnisleistungen erfüllt sein? Welche exekutiven Anforderungen gewährleisten, dass im interaktiven Prozess und in einem entsprechenden situativen Kontext Intersubjektivität und Verständigung zwischen Kommunikationspartnern hergestellt werden können [1]? Schon bei diesen Fragen zeigt sich angesichts einer außerordentlich großen Vielfalt möglicher kognitiver Symptome, Schweregradabstufungen und potenzieller kombinatorischer Muster, um welch heterogene Phänomene es geht, wenn die Spezifik kognitiver Kommunikationsstörungen erfasst und hinsichtlich der Mög- lichkeiten therapeutischer Interventionen interpretiert werden soll $[2,3]$.

Um in diesen interaktiven Prozessen Sinneinheiten einer stringenten thematischen Entwicklung von Gedankengängen zuordnen zu können [4], muss Relevantes von Irrelevantem unterschieden werden. Darüber hinaus müssen Kohärenz und Eindeutigkeit hergestellt werden können. Andernfalls kommt es zu irritierenden Abschweifungen und kausal schwer nachvollziehbaren Strukturen. Zusätzlich ist die Fähigkeit zur Abstraktion und zur Einbeziehung der Perspektive des Gegenübers im situativen Kontext erforderlich (Theory of Mind). Das Kommunikationsverhalten der Interaktionspartner ist zu unterschiedlichen kommunikativen Anlässen an einer Vielzahl konventionalisierter Vorgaben (z. B. sozialen Verhaltensnormen, Scripts, Frames) orientiert. Durch diese wird die Sinnhaftigkeit kommunikativer Handlungen in Kontexten wechselseitig nachvollziehbarer und für die Beteiligten verständlich. Auch nonverbale Handlungen haben einen wichtigen Einfluss auf diesen Verständigungsprozess.

In diesen Prozessen arbeiten im Normalfall logisch-kausale Operationen mit lexikalischen und syntaktischen Mitteln einander zu, wenn etwa kohärente Verknüpfungen durch sprachliche Kohäsionsmittel gekennzeichnet werden (vgl. z. B. logische Subjunktionen wie „weil“ oder adverbiale Kennzeichnungen eines Widerspruchs wie „allerdings“). Wenn das aufgrund kognitiver und/oder sprachlicher Symptome nicht ungestört gelingt, kann die kognitiv-propositionale Struktur von Äußerungen für den Kommunikationspartner unverständlich bleiben. Bei Patienten mit kognitiven Kommunikationstörungen beobachten wir zudem Auffälligkeiten bei ihrer Wahl des stilistischen Registers; ihre 
Äußerungen können dabei ausgesprochen elaboriert, artifiziell und im konkreten sozialen Anlass irritierend wirken.

Ebenso können Missverständnisse entstehen, wenn Äußerungen z. B. bei semantischen Symptomen vage oder inhaltsleer bleiben oder nicht angemessen korrigiert werden können. Wir beobachten auch vorschnelle und teilweise unkontrolliert wortreiche Äußerungen, die oft ebenso schwer nachvollziehbar sind wie stark reduzierte Äußerungen bei ausgeprägten Antriebsstörungen. Die skizzierten Symptome können eine erfolgreiche Kommunikation besonders im Zusammenhang mit oft vorliegenden Einschränkungen des Störungsbewusstseins (Anosognosie) beeinträchtigen.

\footnotetext{
Merke

Die Heterogenität der Störungsprofile und die häufig nur diskrete Ausprägung von Symptomen bei kognitiven Kommunikationsstörungen erfordern eine systematische Berücksichtigung von Selbst- und Fremdeinschätzungen.
}

Aber nicht nur die große Vielfalt und Heterogenität von Störungsprofilen stellen eine große diagnostische Herausforderung dar. Besonders auch die gelegentlich nur diskret ausgeprägten Symptome können klinisch schwer einzuordnen sein. Sie können kommunikative Prozesse durchaus erheblich stören und sind als pathologische Phänomene dringend von prämorbiden Eigenheiten des individuellen Kommunikationsverhaltens von Probanden abzugrenzen. Daher schlagen die Autoren des vorliegenden Beitrags vor, sich den zur Disposition stehenden kommunikativen Handlungen aus unterschiedlichen Perspektiven zu nähern. Dazu sollen baukastenartig geeignete Screenings und Rating-Verfahren zur Beurteilung verschiedener Parameter des kommunikativen Handelns einbezogen werden. Unter anderem sollen über geeignete Fragebögen Einschätzungen der Patienten ihres eigenen Kommunikationsverhaltens, die Beurteilungen der Angehörigen und die der Therapeuten erhoben und therapeutischen Entscheidungen (auch vor und nach Therapie) zugrundegelegt werden.

Im Folgenden sollen dieser gleichsam multidimensionale Zugang zu kognitiven Kommunikationsstörungen und sein Gewinn für die Entwicklung sinnvoller Therapieziele demonstriert werden. Dazu werden das Experten-Rating BEKOS zur Bewertung kommunikativer Leistungen und Sprache [5] sowie das etablierte Verfahren zur Selbst- und Fremdbewertung LCQ, der La Trobe Questionnaire [6], beschrieben. Anschließend werden sie zusammen auf ein konkretes Fallbeispiel angewendet.

\section{Rating-Verfahren \\ Bewertung kommunikativer Leistungen und Sprache (BEKOS)}

Das Ziel dieses Verfahrens ist es, auf der kommunikativen Oberfläche therapierelevante Symptome kognitiver Kommunikationsstörungen unabhängig von der Ätiologie und dem Läsionsort inhaltlich valide und sensitiv zu erfassen. Des Weiteren sollen die Durchführung und Auswertung durch eine standardisierte Datenerhebung und ein Auswertungsmanual objektiviert werden. Das Verfahren erhebt nicht den Anspruch, zwischen ungestörter und gestörter Kommunikation zu unterscheiden. Die Bewertung sieht jedoch eine Schweregradeinschätzung einzelner Symptome vor, um auch die Evaluation von individuellen Therapiefortschritten zu ermöglichen.

\section{Aufbau}

Die Grundlage für das Rating bildet ein ca. 20-minütiges, möglichst natürliches Gespräch über Freizeit, Beruf, Familie, Politik oder andere für den Patienten interessante und emotional ansprechende Themen. Dieses Gespräch wird auf Video aufgezeichnet. Die standardisierte Datenerhebung ist methodisch notwendig, macht jedoch eine Ergänzung durch Fremd- und Selbsteinschätzungen zwingend erforderlich, weil

- leichte Auffälligkeiten ggf. von Angehörigen als prämorbides Verhalten beschrieben werden und

- Gespräche in der Klinik hinsichtlich der Emotionalität, der Auswahl der Gesprächspartner und der situativen Kontexte immer nur bedingt repräsentativ sein können und der Patient in anderen Situationen ein größeres Verhaltensspektrum zeigen kann.

Die BEKOS beinhaltet 67 Items, mit denen sprachliche, sprechmotorische, inhaltliche und behaviorale Dimensionen kommunikativen Handelns auf einer 4-stufigen verbalen Skala („nie“ bis "fast immer/immer“) eingeschätzt werden. Beispielhaft seien an dieser Stelle semantische Vagheit, auffällige Prosodie, Weitschweifigkeit oder auffällige Mimik bzw. Gestik genannt. Die ausgewählten Items wurden in der Literatur als häufige Ursachen für das Scheitern kommunikativer Handlungen thematisiert $[2,7,8]$. Die 67 Items wurden dann unter 12 Hauptmerkmale subsumiert, die als konstitutiv für kommunikatives Handeln gelten. Beispiele dafür sind die Dimensionen Lexikon, Syntax und Kohäsion, nonverbale Kommunikation, Informationsauswahl, soziale Konventionen oder Gesprächsorganisation. 


\section{Validität}

In 2 Vorstudien mit insgesamt 29 Patienten nach Schädel-Hirn-Trauma zeigten sich zum einen gute Beurteilerübereinstimmungen für die Hauptmerkmale $[9,10]$. Zum anderen wurden hypothesenkonforme Korrelationen zwischen exekutiven Dysfunktionen einerseits und den Verhaltensskalen sowie den Skalen zur inhaltlichthematischen Planung gefunden und als Hinweise auf eine gute inhaltliche Validität gewertet.

\section{Merke}

Das Experten-Rating BEKOS ermöglicht eine standardisierte und valide Beschreibung von sprachlichen, inhaltlichen und behavioralen Aspekten kommunikativen Handelns.

\section{La Trobe Questionnaire (LCQ)}

\section{Aufbau}

Beim LCQ handelt es sich um einen Fragebogen, mit dem sowohl die Ausprägung und Häufigkeit von Symptomen als auch Veränderungen in der Kommunikationsfähigkeit vor und nach Erkrankungsbeginn erfasst werden können. Er wurde von Douglas und Kolleginnen [6] entwickelt, um Veränderungen im Gesprächsverhalten nach einem Schädel-Hirn-Trauma sowohl aus der Perspektive der Betroffenen als auch aus der von Angehörigen einschätzen zu können [11,12]. Daher liegen mit an sich gleichen Fragen eine Version zur Selbstbeurteilung (LCQ-S) und eine für die Beurteilung der Kommunikation durch Angehörige (LCQ-A) vor. Die Beurteilung erfolgt anhand einer modifizierten LikertSkala mit 4 möglichen Antwortniveaus, die von „nie/ selten“ (1) bis „für gewöhnlich/immer“ (4) reichen.

Der LCQ besteht aus 30 Fragen, die verschiedene Komponenten der Kommunikationsfähigkeit erfassen. Die theoretische Konstruktion des Fragebogens orientiert sich an Damicos Clinical-Discourse-analysis [13], die von den Kooperationsprinzipien nach Grice [14] abgeleitet ist. Zusätzlich wurden Fragen formuliert, die nach der Forschungsliteratur in Verbindung mit kogni- tiven Defiziten nach Schädel-Hirn-Trauma stehen können wie z.B. Gedächtnis- oder Aufmerksamkeitsdefizite [1].

\section{Auswertung}

Der Gesamt-Score reicht von 30-120 Punkten. Dabei drückt eine höhere Punktzahl ein stärker eingeschätztes Defizit in der Kommunikationsfähigkeit aus (Komponente d3 der ICF [Internationale Klassifikation der Funktionsfähigkeit, Behinderung und Gesundheit]). Der LCQ bietet keinen Cut-off-Wert, mit dem kognitive Kommunikationsstörungen aus Sicht des Fachpersonals diagnostiziert werden können. Er ist als klientenzentriertes Rating vielmehr dazu geeignet, durch den Vergleich der unterschiedlichen Perspektiven in den Fragebögen Abweichungen in der Selbst- und Fremdeinschätzung zu erkennen und das jeweils beobachtete Defizit quantifizierbar abzubilden. Darüber hinaus können neben den Diskrepanzen zwischen Selbst- und Fremdeinschätzung auch jene Bereiche identifiziert werden, die für den Betroffenen selbst das größte Partizipationsdefizit darstellen. Hilfreich ist die Zuordnung von Fragen zu den verschiedenen Dimensionen des Fragebogens (wie Enthemmung bzw. Impulsivität; - Tab.1). Damit ist die Eingrenzung auf solche Bereiche möglich, die besonders beeinträchtigt sind.

\section{Validität}

Der LCQ wurde seit 2000 von unterschiedlichen Forschergruppen mit verschiedenen Populationen in Bezug auf seine psychometrischen Eigenschaften eingehend untersucht $[11,12,15]$. Für den Erwachsenenbereich liegen Normdaten von 18-65 Jahren vor. Eine Faktorenanalyse ergab für die 4 ermittelten Dimensionen (vgl. > Tab. 1) eine sehr gute interne Konsistenz.

\section{Merke}

Das Verfahren LCQ beschreibt Veränderungen im Gesprächsverhalten nach einem Schädel-Hirn-Trauma sowohl aus der Perspektive der Betroffenen als auch aus der von Angehörigen.

- Tab. 1 Dimensionen und Fragebeispiele des LCQ (Quellen: Büttner J, Klingenberg G, Schmid K. Der La Trobe Communication Questionnaire. Unveröffentlichtes Material. Bad Aibling: Schön Klinik Bad Aibling; 2016 und Struchen MA, Pappadis MR, Mazzei DK et al. Perceptions of communication abilities for persons with traumatic brain injury: validity of the La Trobe Communication Questionnaire. Brain Injury 2008; 22 [12]: 940-951).

\begin{tabular}{|l|l|}
\hline Dimensionen & Fragenbeispiele \\
\hline Initiieren bzw. Gesprächsfluss & 18. Hat er/sie Schwierigkeiten, ein Gespräch zu beginnen? \\
\hline Enthemmung bzw. Impulsivität & 12. Wird er/sie von Nebensächlichkeiten im Gespräch abgelenkt? \\
\hline kommunikatives Gelingen bzw. Wirksamkeit & 19. Kann er/sie den Überblick über Hauptinhalte des Gesprächs behalten? \\
\hline Partnersensitivität & 4.Wechselt er/sie zu schnell zu einem anderen Gesprächsthema? \\
\hline
\end{tabular}




\section{Einsatz der Rating-Verfahren in Diagnostik und Therapieplanung}

\section{FALLBEISPIEL}

Der Patient H. K. (27) erlitt 2018 ein schweres Schädel-Hirn-Trauma mit einer traumatischen Subarachnoidalblutung links-frontal, Mikroblutungen mit diffusen axonalen Schädigungen sowie einer Kalottenfraktur links-frontal. H. K. lebte in einer Wohngemeinschaft und absolvierte eine Ausbildung zum Kosmetiker mit dem Ziel eines anschließenden Studiums der Kosmetiktechnologie. Zuvor wurden 4 Ausbildungsversuche und schulische Weiterqualifikationen abgebrochen.

Fünf Monate nach dem Ereignis waren noch eine reduzierte Belastbarkeit, eine erhöhte interne Ablenkbarkeit sowie der teilweise Verlust handlungsleitender Ziele zu beobachten. Zwölf Monate nach dem Ereignis erfolgte eine erneute Diagnostik und Behandlung in der Klinik für Kognitive Neurologie des Universitätsklinikums Leipzig. Dort waren die psychometrischen Tests in den Bereichen der neuropsychologischen und sprachtherapeutischen Leistungen (Aachener Aphasietest, Bielefelder Wortfindungs-Screening, MAKRO [Screening zur Verarbeitung der Makrostruktur von Texten bei neurologischen Patienten]) weitgehend unauffällig. Dennoch fielen im durchgeführten LCQ sehr kritische Selbsteinschätzungen auf, die die Mutter des Patienten nur teilweise bestätigte. Wenn Selbst- und Fremdeinschätzungen nicht übereinstimmen, sollte das Experten-Rating die Symptome konkretisieren und für alle Beteiligten verständlich erläutern: Mithilfe der BEKOS wurde ein differenzierteres Leistungsprofil erstellt, sodass letztlich aus der Kombination der 3 Beurteilerperspektiven alltagsrelevante und spezifische Therapieziele festgelegt werden konnten. Sprachliche Probleme auf Wort- oder Satzebene wurden zwar genannt, aber letztlich als wenig relevant eingeschätzt ( $\triangleright$ Tab. 2). Als eine zentrale Einschränkung wurde in den Selbst- und Fremdeinschätzungen von H. K.s kommunikativen Handlungen gesehen, dass von ihm geäußerte Gedanken nicht immer einem logisch nachvollziehbaren Zusammenhang zugeordnet werden konnten. Dies wurde auch in den Beurteilungen in der BEKOS bestätigt. Allerdings wurde dieses Symptom in der Experteneinschätzung anders als von der Mutter im Zusammenhang mit seiner gedanklichen Sprunghaftigkeit und Weitschweifigkeit begründet. Erschwerend für die Kohärenzbildung beschrieb die BEKOS noch unmotivierte pseudointellektuelle Ausführungen und Analogien sowie längere, überzogen egozentrische Episoden. In den beiden LCQ-Bewertungen wurde außerdem der mangelnde Überblick über die Hauptinhalte genannt. Diese Einschätzung wurde in der BEKOS differenzierter beschrieben, denn H. K. war durchaus in der Lage, das Hauptthema oder thematische Nebenstränge zu überblicken. Dennoch waren unzureichende Relevanzgewichtungen, assoziative Einschübe und Bezugnahmen auf Nebensächlichkeiten auffällig. H. K. fehlte also offenbar nicht der Überblick über die thematische Entwicklung, sondern er bezog sein Wissen darüber in die Planung seiner Gesprächsbeiträge nur unangemessen ein. Diese Dissoziation zwischen Wissen und Handeln wird als eine typische Verhaltensauffälligkeit im Rahmen exekutiver Dysfunktionen beschrieben $[2,16]$. In den Fremdeinschätzungen der Mutter waren in H. K.s Äußerungen darüber hinaus häufig ein unangemessener Sprechstil und eine auffällige Wortwahl zu beobachten. Das kam in der Selbsteinschätzung des Patienten so nicht vor. Nach der Experteneinschätzung der BEKOS handelte es sich dabei um ein komplexes Verhaltensmuster, bei dem H. K. zu einem häufigen Wechsel zwischen überförmlichen und sehr legeren Handlungen neigte. Dabei wurde sein Hang zu einem elaborierten Sprachstil mit vielen Fremdwörtern deutlich. H. K. bestätigte dieses Verhalten zwar in seiner Selbsteinschätzung, allerdings gab seine Mutter eine starke Akzentuierung dieses prämorbiden Verhaltens an. Dieser Hinweis wurde in die Therapiezieldefinitionen einbezogen. Für die Festlegung der globalen Therapieziele war zunächst der LCQ maßgeblich, die spezifischen Therapieschwerpunkte wurden dann durch die differenzierten Einschätzungen in der BEKOS spezifiziert:

- 1.Ziel: Der Patient sollte daran arbeiten, dass seine Äußerungen in den gegebenen Kontexten nachvollziehbar sind und er sollte assoziative Sprünge, pseudointellektuelle Ausführungen sowie ichbezogene Episoden vermeiden.

- 2.Ziel: Der Patient sollte seinen partiell gekünstelten Sprachstil und die teilweise unangemessene Wortwahl besser wahrnehmen und kontrollieren und die übertriebene Verwendung von Fremdwörtern und elaboriertem Code reduzieren. Darüber hinaus sollte er seine Rückmeldungen übergroßer Förmlichkeiten vermeiden und von seinem teilweise unangemessen legeren Verhalten absehen.

Zusammenfassend konnten mithilfe des LCQ alltagsrelevante Auffälligkeiten identifiziert und anhand der Kategorien des BEKOS ausdifferenziert werden, sodass relevante und nachvollziehbare Therapieziele definiert werden konnten. 
- Tab.2 Zusammenfassung der Einschätzungen und abgeleitete Therapieschwerpunkte: Gegenüberstellung relevanter Symptome in den Verfahren.

\begin{tabular}{|c|c|c|}
\hline LCQ Selbsteinschätzung & LCQ Mutter & BEKOS-Experte \\
\hline vage Wörter, Wortfindungsstörungen & 0 & $\sqrt{ }$ vereinzelt Vagheit \\
\hline zu langes Verharren bei Themen & 0 & 0 \\
\hline zu schnelles Wechseln zu anderen Themen & 0 & $\sqrt{ }$ sprunghaft, thematisch unpassend, weitschweifig \\
\hline im Gespräch von Nebensächlichkeiten abgelenkt & 0 & $\sqrt{ }$ assoziativ, sprunghaft \\
\hline Auslassungen wichtiger Details & 0 & $\sqrt{ }$ \\
\hline $\begin{array}{l}\text { Probleme, Gedanken in logischen Zusammenhang } \\
\text { zu bringen }\end{array}$ & $\sqrt{ }$ & $\begin{array}{l}\sqrt{ } \text { unpassende } \text { Inhalte }^{11} \text {, assoziativ }{ }^{11}, \text { weitschweifig }{ }^{1)} \text {, } \\
\text { Auslassungen }{ }^{1)}\end{array}$ \\
\hline Probleme, Hauptinhalte zu überblicken & $\sqrt{ }$ & 0 \\
\hline unangemessen in Wortwahl und Sprechstil & 0 & $\begin{array}{l}\sqrt{ } \text { übertriebene Prosodie, laut, viele Fremdwörter }{ }^{1)} \text {, } \\
\text { elaboriert }{ }^{1)} \text {, teils überförmlich'1), teils leger }{ }^{1)}\end{array}$ \\
\hline $\begin{array}{l}\text { 1) primäre Therapieschwerpunkte. } \\
0=\text { Symptom nicht bestätigt } \\
\sqrt{=} \text { Symptom bestätigt }\end{array}$ & & \\
\hline
\end{tabular}

\section{FAZIT}

Die Autoren des vorliegenden Beitrags haben im Fallbeispiel anhand der unterschiedlichen Beobachtungen demonstrieren können, dass kommunikative Handlungen des Patienten H. K., die in eingeführten neurolinguistischen und sprachtherapeutischen Tests weitgehend unauffällig blieben, in den Einschätzungen konkreten Kommunikationsverhaltens in der BEKOS und nach den verschieden bewerteten Beobachtungen im LCQ

- als relevante Kommunikationsstörungen identifiziert,

- in Begründungszusammenhänge eingeordnet und

- gewinnbringend für konstruktive therapeutische Entscheidungen verwendet werden konnten.

Die ICF-Ebene der Partizipation setzt die Beschreibung alltagsrelevanter Defizite durch die Betroffenen und Angehörigen voraus.
Dabei ergänzten sich die Einschätzungen aus unterschiedlichen Perspektiven, weil etwa der Betroffene seine Wahrnehmungen im Prozess der Krankheitsbewältigung anders einordnete als die klinischen Experten. Zusätzlich konnten die Beobachtungen der Angehörigen auf allgemeiner Ebene durch die Experteneinschätzungen in der BEKOS detailliert spezifiziert werden. Dadurch wurde dem Umstand besser Rechnung getragen, dass das Patientenverhalten im klinischen Kontext in der Regel anders ausgeprägt sein kann als im häuslichen Umfeld, wo beispielsweise andere Rollenkonstellationen und verschiedene Kontrollroutinen (z. B. die Impulskontrolle) wirksam sein können. Dadurch waren die Autoren bei allen nachfolgenden therapeutischen Überlegungen in der Lage, die ICF-Ebene der Partizipation [17] besser abzubilden.

\section{Schlüsselwörter}

Kognitive Kommunikationsstörungen, Rating-Verfahren, Selbst- und Fremdeinschätzung, Therapieplanung

\section{Interessenkonflikt}

Die Autorinnen/Autoren geben an, dass kein Interessenkonflikt besteht.

\section{Autorinnen/Autoren}

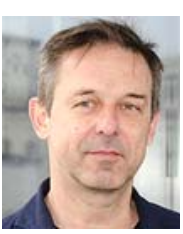
tung, kognitive Kommunikationsstörungen, Wortverarbeitung und computerunterstütztes Lernen bei Aphasie. 


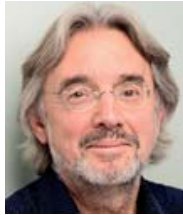

\section{Ralf Glindemann ${ }^{2}$}

Dr. phil.; Neurolinguist, klinischer Linguist. Wissenschaftlicher Mitarbeiter in der Neurologie an der Uniklinik RWTH Aachen, Arbeitsschwerpunkt: Therapieforschung. Dann über Jahrzehnte Leiter der Neurolinguistik in der Klinik für

Neuropsychologie am Klinikum Bogenhausen. Aktuell Projekte in der Entwicklungsgruppe Klinische Neuropsychologie (EKN) an der LMU München.

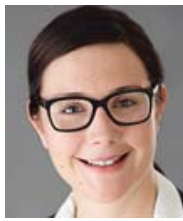

\section{Julia Büttner-Kunert ${ }^{3}$}

Dr. phil.; 2014 Promotion zum Einfluss exekutiver Funktionen auf die Text- und Diskursproduktion bei Personen mit kognitiven Kommunikationsstörungen. Mehr als 12 Jahre klinische Linguistin in der Neurologie mit Spezialisierung auf kognitive Kommunikationsstörungen. Seit 2009 Dozentin für Aphasiologie und Pragmatik an der LMU München, Autorin von Fachbüchern und Screening-Verfahren (MAKRO). Seit 2019 Leiterin eines vom BMBF geförderten Projekts zur Neuropragmatik insbesondere bei demenziellen Erkrankungen und Schädel-Hirn-Trauma.

\section{Institute}

1 Tagesklinik für Kognitive Neurologie, Universitätsklinikum Leipzig

2 Entwicklungsgruppe Klinische Neuropsychologie (EKN), LMU München

3 Fakultät für Sprach- und Literaturwissenschaften, Departement 13 Germanistik, Komparatistik, Nordistik, Deutsch als Fremdsprache/Studiengang Sprachtherapie, LMU München

\section{Korrespondenzadresse}

Frank Regenbrecht

Tagesklinik für Kognitive Neurologie

Liebigstraße 16, Haus 2

04103 Leipzig

E-Mail: frank.regenbrecht@medizin.uni-leipzig.de

\section{Literatur}

[1] Matthes-von Cramon G , von Cramon DY. Störungen exekutiver Funktionen. In: Sturm W, Herrmann M, Wallesch CW Hrsg. Lehrbuch der Klinischen Neuropsychologie. Lisse, NL: Swets \& Zeitlinger; 2000: 392-410

[2] Büttner ], Glindemann R. Kognitive Kommunikationsstörungen. Fortschritte der Neuropsychologie19 Göttingen: Hogrefe; 2019

[3] Regenbrecht F, Guthke T. Kognitive Kommunikationsstörungen in der Sprachtherapie und der Neuropsychologie. Aphasie und Verwandte Gebiete 2017; 1: 16-30

[4] Glindemann R. Wenn Themen sich unkontrolliert entwickeln. Kognitiv-neurolinguistische Überlegungen zu weitschweifigen Äußerungen. Neurolinguistik 2009; 21: 59-99
[5] Regenbrecht F, Schmidt M. Bewertung Kommunikativer Leistungen (BEKOS). Unveröffentlichtes Material. Leipzig: Tagesklinik für Kognitive Neurologie, UKL Leipzig; 2017

[6] Büttner J, Klingenberg G, Schmid K. Der La Trobe Communication Questionnaire. Unveröffentlichtes Material. Bad Aibling: Schön Klinik Bad Aibling; 2016

[7] Linscott R], Knight RG, Godfrey HPD. The Profile of Functional Impairment in Communication (PFIC): a measure of communication impairment for clinical use. Brain Injury 1996; $10: 397-412$

[8] Cummings L. Clinical pragmatics. Cambridge: University Press; 2009: 88-117

[9] Schwarz M, Grewe T, Guthke T et al. Beurteilungsbogen zur Erfassung kommunikativer Leistungen nach Schädelhirntrauma [Masterarbeit Logopädie]. Idstein: Universität Idstein; 2013

[10] Meyer K, Guthke T, Bernhard F. Communication disturbances after traumatic brain injury [Masterarbeit Neuropsychologie]. Strasbourg: Université Strasbourg; 2014

[11] Douglas JM, O'Flaherty CA, Snow PC. Measuring perception of communicative ability: the development and evaluation of the La Trobe communication questionaire. Aphasiology 2000; $14: 251-268$

[12] Douglas JM, O’Flaherty CA, Snow PC. Measuring perceived communicative ability after traumatic brain injury: reliability and discriminant validity of the La Trobe Communication Questionnaire. J Head Trauma Rehab 2007; 22: 31-38

[13] Damico JS. Clinical discourse analysis: a functional approach to language assessment. In: Charlann S ed. Communication skills and classroom success: assessment of language-learning disabled students. San Diego: College-Hill Press; 1985: 165-204

[14] Grice HP. Logic and conversation. In: Cole P, Morgan JL Hrsg. Speech acts (= syntax and semantics. Bd. 3. New York: Academic Press; 1975: 41-58

[15] Struchen MA, Pappadis MR, Mazzei DK et al. Perceptions of communication abilities for persons with traumatic brain injury: validity of the La Trobe Communication Questionnaire. Brain Injury 2008; 22: 940-951

[16] Thöne-Otto A, Schellhorn A, Wenz C. Persönlichkeits-und Verhaltensstörungen nach Hirnschädigung. Göttingen: Hogrefe; 2018

[17] Internationale Klassifikation der Funktionsfähigkeit, Behinderung und Gesundheit (IFC). Deutsches Institut für Medizinische Dokumentation und Information (DIMDI) im Auftrag des Bundesministeriums für Gesundheit (BMG) unter Beteiligung der Arbeitsgruppe ICD des Kuratoriums für Fragen der Klassifikation im Gesundheitswesen (KKG); 2005: (Stand: 24.11.2019) Im Internet: https://www.dimdi.de/ dynamic/de/klassifikationen/downloads/?dir=icf

Bibliografie

DOI https://doi.org/10.1055/a-1043-7259

Online-Publikation: 21.4.2020 | Sprache · Stimme · Gehör 2020; 44: 78-83

(c) Georg Thieme Verlag KG Stuttgart · New York ISSN 0342-0477 